\title{
Comparison of Terrestrial and Lunar Time Scales by Giant Pulsar Impulses
}

\author{
A. E. Rodin ${ }^{a}$ * , V. V. Oreshko ${ }^{a}$, and V. A. Fedorova ${ }^{a}$ \\ ${ }^{a}$ Pushchino Radio Astronomy Observatory, Astro Space Center, Lebedev Physical Institute, \\ Russian Academy of Sciences, Pushchino, 142290 Russia \\ *e-mail: a.e.rodin@mail.ru \\ Received April 30, 2021; revised July 26, 2021; accepted July 27, 2021
}

\begin{abstract}
We have developed a model for the time delay of pulse arrival between stations on the Moon and Earth. Comparison of the lunar and terrestrial time scales is proposed to be carried out by comparing the arrival time moments of giant pulses from pulsars. A method for such a comparison has been developed based on the cross-correlation analysis of the received pulses. Using the example of giant pulses from the pulsar PSR $0531+21$, we showed that the error of comparing scales in the case of a high signal-to-noise ratio reaches a sub-discrete level and, thus, is determined by the reception band of the recording equipment.
\end{abstract}

Keywords: pulsars, time scales, giant pulses

DOI: $10.1134 / \mathrm{S} 1063772921110068$

\section{INTRODUCTION}

The problem of comparison and synchronization with high accuracy of time scales spaced over long distances has been and remains relevant to the present day. On the Earth scale, this problem is solved with the help of an operating satellite navigation system GLONASS, using VLBI methods. At cosmic distances beyond the orbit of the Moon, it is proposed to use a comparison and synchronization of time scales using giant pulses (GP) of a pulsar. This method was proposed in [1].

Giant pulses from pulsars are not suitable for timing and creating time scales, since their appearance inside a pulsar pulse is random, but giant pulses have a very short duration, as a rule, determined by the frequency bandwidth of the recorder, their amplitude and repetition interval are unique in a given time interval, and they have a very high spectral radiation density, in a pulse 4-6 orders of magnitude higher than the average value. Such properties make it possible to use antennas with a relatively small effective area for their registration, which can be deployed on other bodies of the Solar System.

The exploration of the Moon is a priority task for space agencies in many countries, both because of the independent value of the Moon, for example, in terms of mining, and because of the development of space technologies for flights to more distant bodies of the Solar System. In this regard, comparison of time scales is one of the most important parts of radio technical support for interplanetary navigation, communications, and the propagation of precise time signals.
The potential accuracy of comparing time scales using GP is estimated to be $<1$ ns (see below), which is comparable to the accuracy of comparisons using a Global Navigation Satellite Systems (GNSS) on the Earth.

The main purpose of this paper is to demonstrate the capabilities of the GP time scale synchronization technology using the example of comparing the lunar and terrestrial time scales. For this purpose, in the paper we developed a model of the delay between stations on the Moon and the Earth, presented an algorithm for comparing scales by giant impulses based on their cross-correlation, and gave an estimate of the accuracy of comparing scales.

\section{PROPERTIES OF GIANT PULSES AND PECULIARITIES OF THEIR OBSERVATIONS}

Let us single out separately the pulsars emitting giant radio pulses, the peak flux density of which can exceed the peak flux density of average pulses from several tens to hundreds of thousands of times. The main properties of GP are [2-4]:

- anomalously high peak radio flux density (for the pulsar in the Crab Nebula B0531+21 at frequencies of 600-1400 MHz - up to several million Jansky;

- short duration (from several nanoseconds to several microseconds);

- power law of distribution by peak flux density/pulse energy;

- high directivity of radiation, which follows from the narrow longitudinal localization of the GP; and 
- high degree of GP polarization.

The total number of known pulsars with GP is 16 [5], including 11 normal and 5 millisecond pulsars, including the "classical" representatives: the pulsar in the Crab Nebula B0531 $+21(P=33 \mathrm{~ms})$ and the single millisecond pulsar B1937+21 $(P=1.56 \mathrm{~ms})$, as well as a number of pulsars observed only at low frequencies and having a peak flux density of up to several hundred Jansky.

The individual pulses of the pulsar PSR 0531+21 in the Crab Nebula consist entirely of short (ordinary and giant) pulses. The distribution of their amplitudes corresponds to a power law. The frequency of the appearance of especially powerful GPs of this pulsar was estimated as a result of its long-term observations with the RT-64 radio telescope in Kalyazin at frequencies of $600 \mathrm{MHz}$ and $4.85 \mathrm{GHz}$. At a frequency of $600 \mathrm{MHz}, 6-8 \mathrm{GPs}$ with a peak flux density $>300 \mathrm{kJy}$ were recorded for an hour, at a frequency of $4.85 \mathrm{GHz}$, 2-3 GPs were recorded with a flux $>10 \mathrm{kJy}$ [6]. The shortest GP duration was recorded with the Arecibo radio telescope at a frequency of $9.25 \mathrm{GHz}$ in the $2.5 \mathrm{GHz}$ band from the pulsar $0531+21$ in the Crab Nebula [2]. The width of the most powerful detail of the pulse microstructure was $0.4 \mathrm{~ns}$, and the peak flux density was $2.2 \mathrm{MJy}$. Note that the observed microstructure detail is not resolved and its width is limited by the sampling rate (inverse to the frequency band) of the receiving system. That is, in this case, when correlating the GP taken at different points, we use the VLBI method, devoid of its main drawback-period uncertainty. In addition, there is no need to transmit the entire array of observation data over a long interval to the processing center from the spacecraft; it is enough to transmit data at the interval of the GP appearance, which reduces the amount of transmitted data by orders of magnitude. In the case of observations of the pulsar GP in the $400 \mathrm{MHz}$ range with a $50 \mathrm{MHz}$ recording bandwidth, the accuracy of comparing the time scales by the pulsar GP will be better than $10 \mathrm{~ns}$. If we accept a very conservative estimate of the signal-to-noise ratio of $\sim 10$, then the synchronization error will be on the order of $1 \mathrm{~ns}$. If the predetector method of compensating for the dispersive pulse delay in the interstellar medium is implemented on board the spacecraft, the volume of data transmitted from the spacecraft in a communication session can be limited to $20 \mathrm{Mb}$.

\section{MODEL OF THE TIME DELAY BETWEEN OBSERVERS ON THE MOON AND EARTH}

The binding of two time scales is based on measuring the difference in the arrival time of short pulses relative to these scales. As already mentioned, in the role of short duration pulses, it was earlier proposed in [1] to use giant pulses from pulsars. The power of these pulses is such that they can be recorded by antennas of a relatively small area.

For the correct binding of the scales, it is necessary to develop a delay model that will accurately calculate the propagation time between two receiving antennas located on the Moon and Earth. The delay is calculated in several stages (see Fig. 1):

(1) The vector of the mass center of the EarthMoon system is calculated relative to the barycenter of the Solar System $\overrightarrow{B G}(t)$ at the required time moment $t$. For this, modern planetary ephemeris EMP2XXX (Institute of Applied Astronomy, Russian Academy of Sciences) or DE4XX (JPL) are used. The procedure for calculating the coordinates of the Earth-Moon barycenter is called with the parameter NPL $=3$.

(2) The position of the Moon relative to the geocenter $\overrightarrow{T L}(t)$ is calculated. This is also done using ephemeris with NPL $=11$.

(3) With the calculated components of the vectors $\overrightarrow{B G}(t)$ and $\overrightarrow{T L}(t)$, the vectors $\overrightarrow{G L}(t)$ and $\overrightarrow{G T}(t)$ are calculated using the following formulas:

$$
\begin{aligned}
\overrightarrow{G L}(t) & =\frac{m_{T}}{m_{L}+m_{T}} \overrightarrow{T L}(t), \\
\overrightarrow{G T}(t) & =-\frac{m_{L}}{m_{L}+m_{T}} \overrightarrow{T L}(t),
\end{aligned}
$$

where $m_{L}$ and $m_{T}$ are the masses of the Moon and the Earth, respectively.

(4) The vector of the observer position on the Moon $\overrightarrow{L R}(t)$ is determined through rotations by the libration angles, which, in turn, are also calculated from the ephemeris with the parameter NPL $=13$.

$$
\overrightarrow{L R}(t)=\left(\mathbf{R}_{z}[\Psi(t)] \mathbf{R}_{x}[\boldsymbol{\Theta}(t)] \mathbf{R}_{z}[\Phi(t)]\right)^{-1}\left(\begin{array}{c}
x \\
y \\
z
\end{array}\right),
$$

where $x, y$, and $z$ are the coordinates of the radio telescope on the lunar surface in the selenocentric coordinate system defined relative to the principal axes of inertia of the Moon; $\boldsymbol{\Psi}(t), \boldsymbol{\Theta}(t)$, and $\boldsymbol{\Phi}(t)$ are the libration angles; and $\mathbf{R}_{x}$ and $\mathbf{R}_{z}$ are the rotation matrices around the $x$ and $z$ axes, respectively.

(5) The vector of the observer's position on the Earth $\overrightarrow{T O}(t)$ is calculated through a series of successive rotations of the observer's vector in the ITRF system, considering the daily rotation of the Earth, precession, nutation and motion of the pole:

$$
\overrightarrow{T O}(t)=\mathbf{P}^{-1} \mathbf{N}^{-1} \mathbf{R}_{z}(-\theta) \mathbf{R}_{x}\left(y_{p}\right) \mathbf{R}_{y}\left(x_{p}\right)\left(\begin{array}{c}
u \\
v \\
w
\end{array}\right),
$$

where $u, v$, and $w$ are the coordinates of an Earth observer in the ITRF system; $\mathbf{P}$ and $\mathbf{N}$ are the preces- 


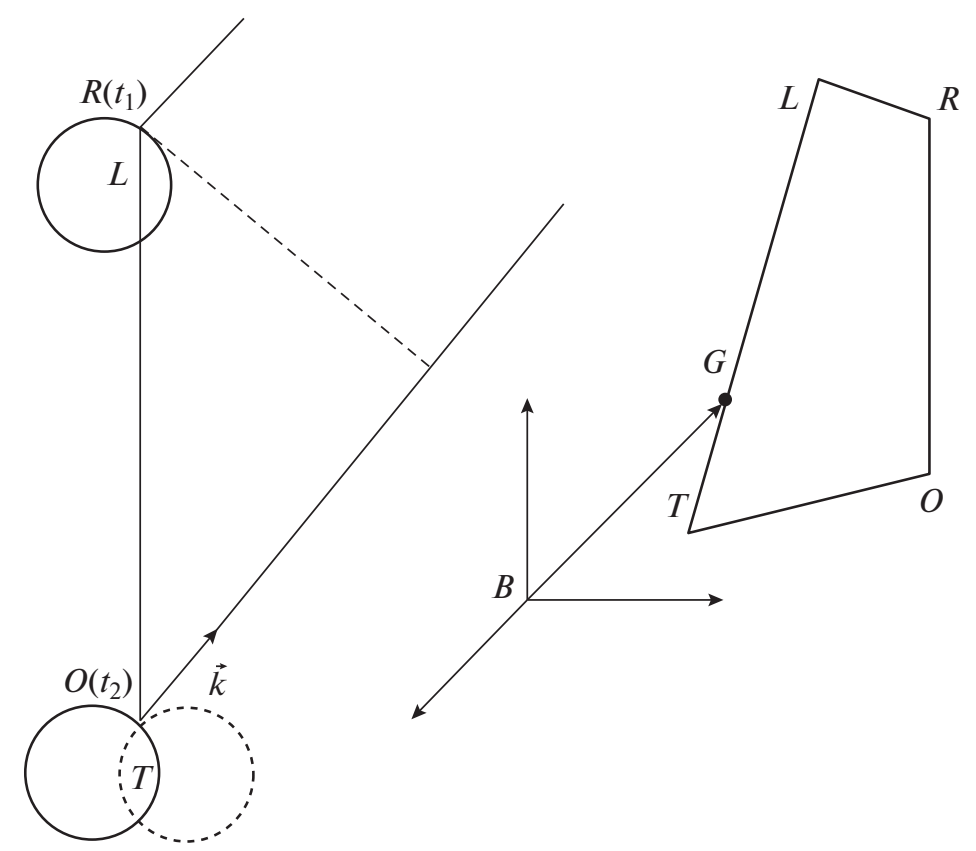

Fig. 1. Mutual geometry of the location of observers on the Moon and Earth. On the left side of the figure, the Moon $L$ and the Earth $T$ are conventionally depicted. The dashed circle shows the position of the Earth at time $t_{1}$. By the time $t_{2}$, when the signal, passing from the Moon, reaches the Earth, it will change position, which is shown by the solid circle. The oblique lines show the direction of arrival of the pulse from the pulsar. $\vec{k}$ is the barycentric unit vector in the direction of the pulsar. $B$ is the barycenter of the Solar System, $G$ is the center of mass of the Earth-Moon system, $T$ is the geocenter, $L$ is the center of mass of the Moon, $O$ is the observer on Earth, and $R$ is the observer on the Moon.

sion and nutation matrices; $\mathbf{R}_{x}, \mathbf{R}_{y}$, and $\mathbf{R}_{z}$ are the rotation matrices around the $x, y$, and $z$ axes; $x_{p}, y_{p}$ are the coordinates of the Earth's pole; and $\theta$ is the sidereal time.

(6) Barycentric vectors of observers $\overrightarrow{B O}$ and $\overrightarrow{B R}$ are calculated as the corresponding sums

$$
\begin{aligned}
& \overrightarrow{B O}=\overrightarrow{B G}+\overrightarrow{G T}+\overrightarrow{T O}, \\
& \overrightarrow{B R}=\overrightarrow{B G}+\overrightarrow{G L}+\overrightarrow{L R} .
\end{aligned}
$$

(7) The time of signal propagation along the direction to the pulsar is calculated (Röhmer's delay):

$$
\Delta t_{r}=-\frac{(\vec{k} \cdot \vec{r})}{c}
$$

where $\vec{r}$ is the barycentric radius vector of the observer $(\overrightarrow{B O}$ or $\overrightarrow{B R}), \vec{k}$ is the barycentric unit vector in the direction on the pulsar, and $c$ is the speed of light.

(8) The signal delay caused by the finite distance to the pulsar is calculated using the formula:

$$
\Delta t_{s}=\frac{[\vec{k} \times \vec{r}]^{2}}{c R}
$$

where $\vec{r}$ is the barycentric radius vector of the observer $(\overrightarrow{B O}$ or $\overrightarrow{B R}$ ), $R$ is the distance to the pulsar, $\vec{k}$ is the barycentric unit vector in the direction of the pulsar, and $c$ is the speed of light.
(9) The relativistic correction of signal propagation in the gravitational field of bodies of the Solar System is calculated according to the following formula:

$$
\gamma=-2 \sum_{p} R_{g p} \ln \left(r_{p}-\vec{k} \cdot \vec{r}_{p}\right),
$$

where $\vec{k}$ is the barycentric unit vector in the direction of the pulsar, $\vec{r}_{p}$ is the vector "observer-gravitating body" (in our case, these are large planets of the Solar System), and $R_{g p}$ is the gravitational radius of the $p$ th planet, which is calculated by the formula $R_{g p}=\frac{2 G M_{p}}{c^{3}}$. The plot of the relativistic correction is shown in Fig. 2.

(10) The difference between the moments of arrival of an impulse to observers on the Earth and the Moon $\Delta t_{c}$ is calculated by the final formula

$$
\begin{gathered}
\Delta t_{c}=\Delta t_{r}\left(t_{2}\right)+\Delta t_{s}\left(t_{2}\right)+\gamma\left(t_{2}\right) \\
-\left[\Delta t_{r}\left(t_{1}\right)+\Delta t_{s}\left(t_{1}\right)+\gamma\left(t_{1}\right)\right],
\end{gathered}
$$

where the time moments $t_{1}$ an $t_{2}$ are expressed in the time scale TB. The plot of the delay model is shown in Fig. 3.

This completes the algorithm for calculating the difference in the arrival times of the pulsar pulse to the observer on the Earth and the Moon. 


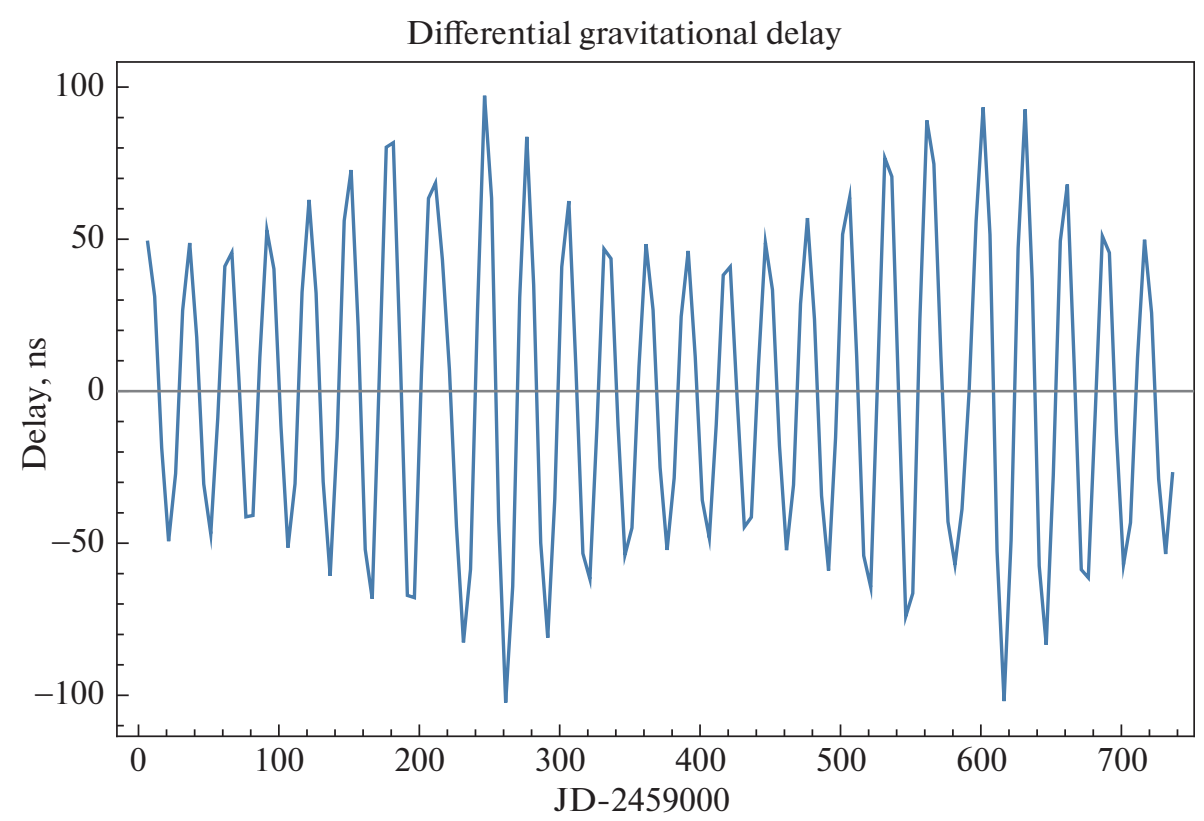

Fig. 2. Model of the differential gravitational delay between the Earth and the Moon ("The Earth minus the Moon"). Variations are visible with a period of one year and with the period of the Moon's revolution.

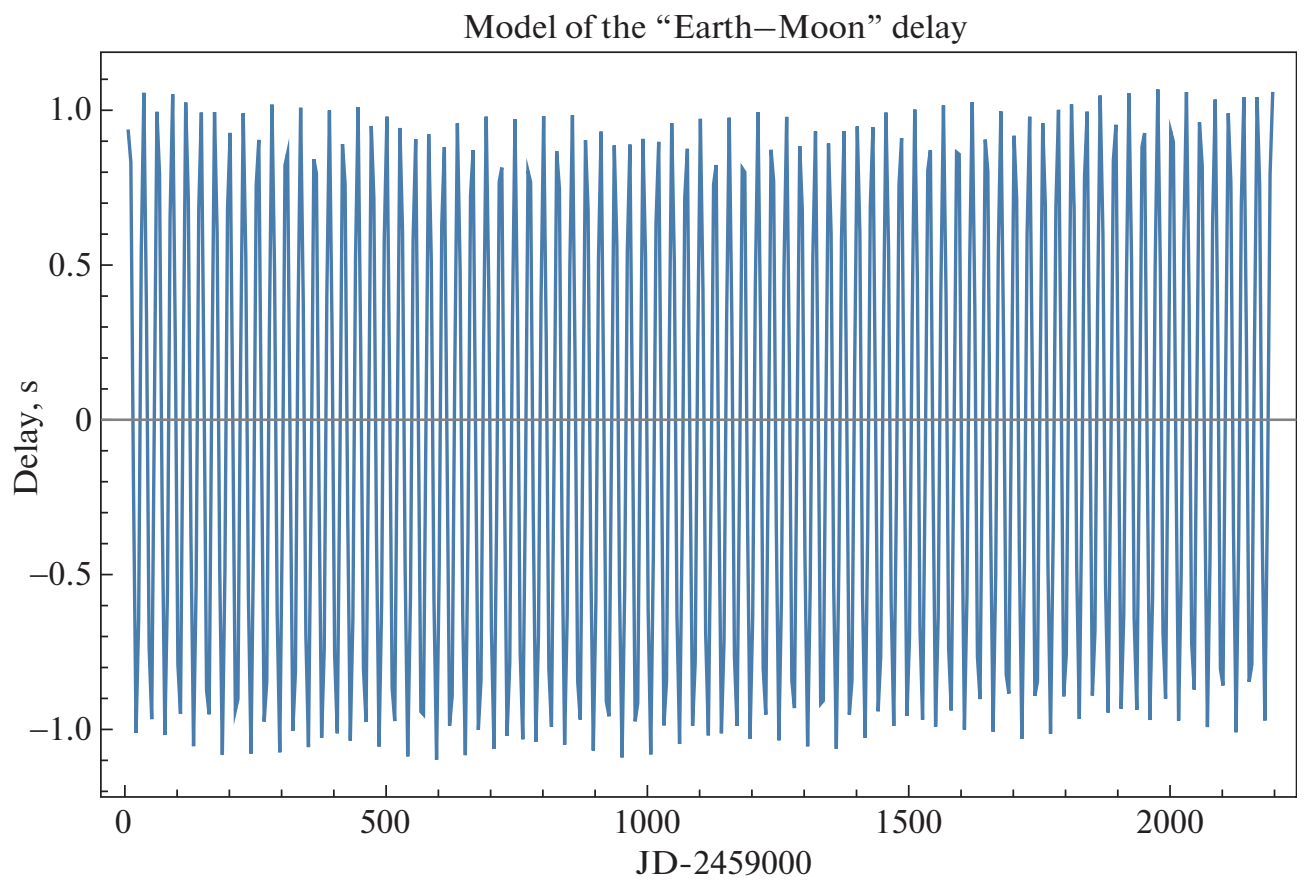

Fig. 3. Model of the "Earth-Moon" delay.

There are a few things to note when using this algorithm:

(1) There is no correction for signal propagation in the interstellar medium. This is due to the fact that when calculating the difference between the moments of arrival of pulses between the Earth and the Moon, these delays are effectively canceled out, since when calculating these delays, the signal propagation path can be considered the same with sufficient accuracy. The only ionized medium between the Earth and the 


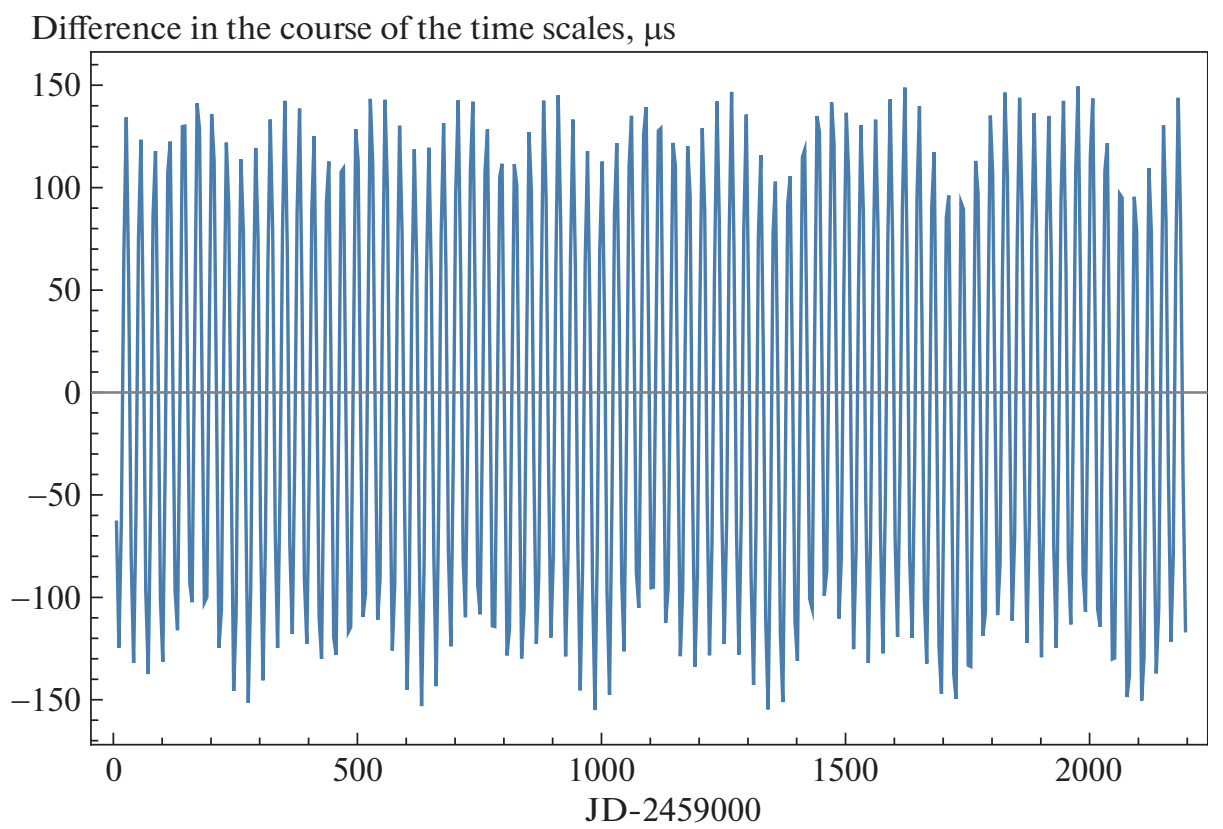

Fig. 4. The difference in the course of the time scales on the Earth and the Moon.

Moon is the Earth's ionosphere, which introduces an additional delay at the $400-1400 \mathrm{MHz}$ observational frequencies of the order of 2-50 ns, which is well within the accuracy of the pulsar timing method. If the measurement accuracy increases in the future, it will not be difficult to calculate the ionospheric correction through the delay at the zenith and considering the oblique path of signal propagation between observers on the Moon and Earth.

(2) It is necessary to consider the different course of the time scales on the Earth and the Moon. In this case, you can follow the same approach as in the TT and TB scales: make the average rate the same, and the difference will be only in the periodic terms. The plot of the mutual motion of the TT scale on the Earth and the scale on the Moon is shown in Fig. 4.

(3) To take into account the light interval (the propagation time of the signal between the Moon and the Earth), the iteration method is used. The process converges very quickly. In practice, one iteration is sufficient.

\section{MODELING THE BINDING OF TWO TIME SCALES USING GIANT PULSES OF THE PULSAR PSR 0531+21}

To carry out the simulation, we used the observations of the pulsar in the Crab Nebula on January 2, 2018 and February 2, 2018. The observations were carried out with the BSA antenna of the Lebedev Physical Institute at a frequency of $111 \mathrm{MHz}$ in a $2.5 \mathrm{MHz}$ band with a temporal resolution of $2.4576 \mathrm{~ms}$. The 6 second files were recorded as shown in Fig. 5.
In [7], the most optimal frequency for observations of pulsars $400-500 \mathrm{MHz}$ was substantiated. To translate observations from 111 to $500 \mathrm{MHz}$, we use the following considerations. With a frequency of $111 \mathrm{MHz}$, pulses from the pulsar PSR B0531+21 show a noticeable exponential scattering, and the scattering in the propagation medium changes with a frequency as $f^{-4}$. Then, to scale this effect, it is enough to change the time scale by $(500 / 111)^{4}=412$ times. Thus, the new sampling interval will be approximately $6 \mu \mathrm{s}$. This will not change the shape of the pulse, but it will change its characteristic width. In the future, it will be convenient to operate precisely with discretes, and lead to units of time at the end of calculations.

To simulate observations of the same pulse by the second antenna on the Moon, the original pulses were shifted by the amount determined by the delay model, and Gaussian white noise with an amplitude equivalent to the resulting signal-to-noise ratio $=3$ and 10 was superimposed on them. Many factors affect the $\mathrm{S} / \mathrm{N}$ ratio. Here, we will assume that it primarily depends on the effective area of the antenna. In the case of a phased array similar to BSA, to obtain the aforementioned $\mathrm{S} / \mathrm{N}$ ratios, the antenna on the Moon should consist of 1.5 or 5 thousand dipoles.

When determining the magnitude of the discrepancy between the scales, the procedure of cross-correlation of pulses was used, followed by smoothing with an exponential pattern (Fig. 7) and inscribing a 4th degree polynomial into the maximum of the crosscorrelation function. It is this procedure that makes it possible to determine the position of the maximum at the sub-discrete level. Cross-correlation functions are 


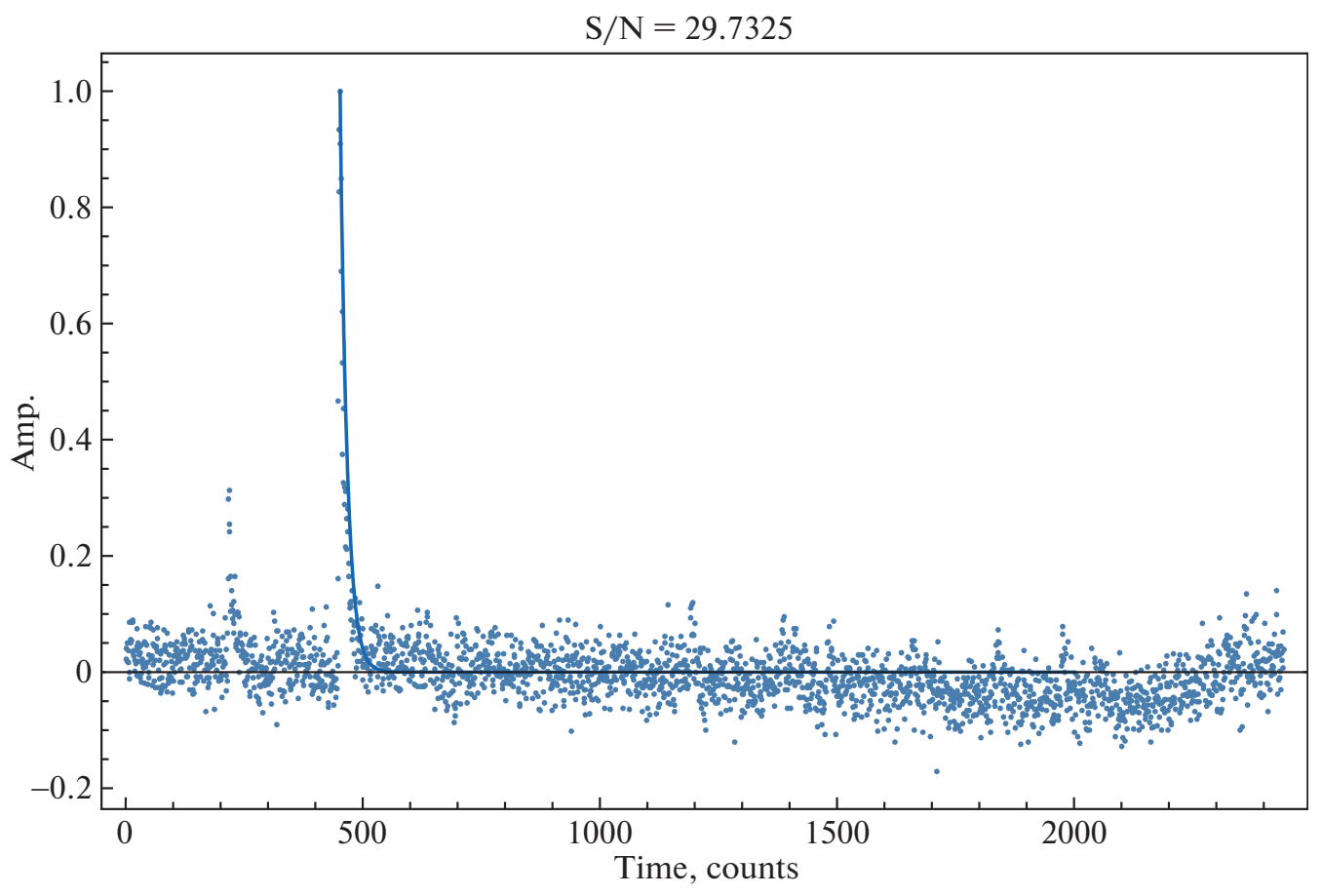

Fig. 5. Giant pulse of the pulsar PSR B0531+21. Observations on January 2, 2018 at 20:11:50.954 (UTC+3).

shown in Fig. 8, and polynomial fitting is shown in Fig. 9.

The results of measuring the binding of time scales are summarized in Table 1, which concludes that the method works even with a relatively weak signal from the Moon $(\mathrm{S} / \mathrm{N}=3)$, since the main signal recorded on Earth confidently "stretches out" the maximum of the cross-correlation function. Let us add that the formal error in measuring the shift does not strongly depend on the signal-to-noise ratio, since the top of the cross-correlation function still remains smooth and is well described by a polynomial, which cannot be said about the actual shift. As expected, it differs more from the model one when the signal-to-noise ratio deteriorates.

Now let us consider the effect of the frequency channel width on the accuracy of measuring the pulse position and, accordingly, measuring the binding of two scales. Let us give the formula for pulse broadening with the measure of dispersion $D M$ in the band $\Delta f$ $[\mathrm{Hz}]$ at the frequency $f[\mathrm{~Hz}]$ :

$$
\Delta t=-\frac{2 D M \Delta f}{k f^{3}}, \quad k=2.410331 \times 10^{-16} .
$$

With the used parameters $f=500 \mathrm{MHz}, B=2.5 \mathrm{MHz}$, the number of channels $=512$ we obtain $\Delta f=$

Table 1. The magnitude of the discrepancy between the two scales, measured at different signal-to-noise ratio of the pulse received on the Moon, different shift and different width of the frequency channels. Values are in microseconds and discrete rate $=6.160618 \mu \mathrm{s}$

\begin{tabular}{c|l|l|c|c|c|c}
\hline Pulse & Model, $\mathrm{s}$ & Observ., $\mathrm{s}$ & Difference, $\mu \mathrm{s}$ & Accuracy, $\mu$ s & Signal-to-noise & $\begin{array}{c}\text { Channel width, } \\
\mathrm{Hz}\end{array}$ \\
\hline 02.01 .2018 & -1.0211252 & -1.0211247 & 0.480 & 0.474 & 10 & 4873 \\
02.02 .2018 & -0.5640922 & -0.5640902 & 1.926 & 0.762 & 10 & 4873 \\
02.01 .2018 & -1.0211252 & -1.0211281 & -2.880 & 0.294 & 3 & 4873 \\
02.02 .2018 & -0.5640922 & -0.5640951 & -2.52 & 0.912 & 3 & 4873 \\
02.01 .2018 & -1.0211252 & -1.0211236 & 4.392 & 0.024 & 10 & 53600 \\
02.02 .2018 & -0.5640922 & -0.5640830 & 9.216 & 0.066 & 10 & 53600 \\
02.01 .2018 & -1.0211252 & -1.0211040 & 21.180 & 0.048 & 3 & 53600 \\
02.02 .2018 & -0.5640922 & -0.5641035 & 11.292 & 0.090 & 3 & 53600 \\
\hline
\end{tabular}




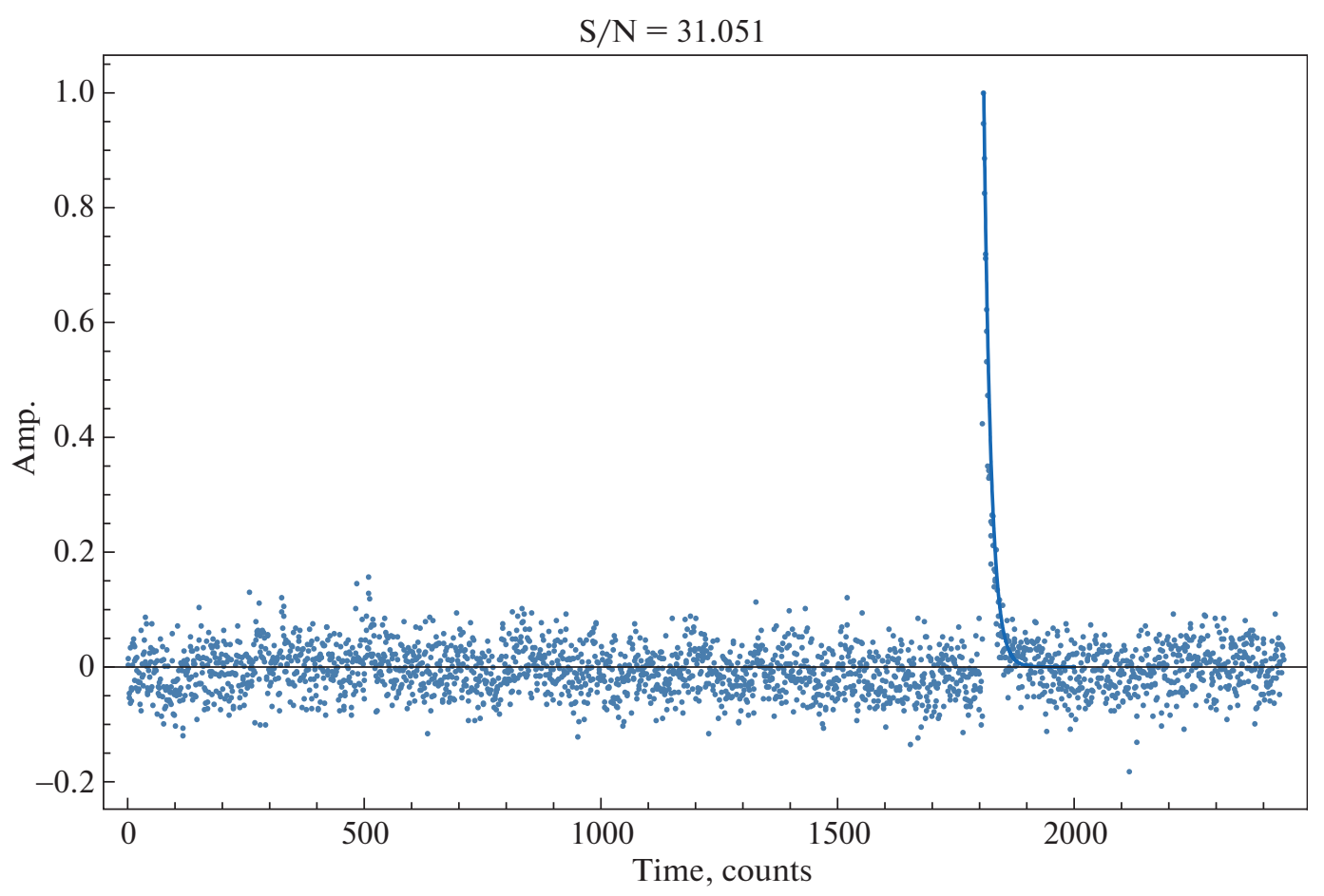

Fig. 6. Giant pulse of the pulsar PSR B0531+21. Observations on February 2, 2018 at 18:09:58.0769 (UTC+3).

$4873 \mathrm{~Hz}$. Substituting these values into formula (1), we obtain the broadening value of $18 \mu \mathrm{s}$, which is equal to 3 discretes.

Furthermore, the simulation was carried out for a channel width of $53.6 \mathrm{kHz}$. From the point of view of mathematics, the use of a wider frequency channel, leading to pulse broadening, is equivalent to convolution of the total pulse $x(\tau)$ with a rectangular core, the width of which is equal to the broadening inside the channel

$$
y(t)=\int_{-\infty}^{\infty} \Pi(t-\tau) x(\tau) d \tau
$$

In the discrete case, the $53.6 \mathrm{kHz}$ channel width is equivalent to a rectangular window with a wide of 11 counts. Convolution with a window of this width was applied to the original pulse.

Also, in the calculations, we used two values of the signal-to-noise ratio $=3$ and 10 . The results of this part of the simulation are shown in the bottom four lines of the Table 1. It is clearly seen from them that the broadening of the pulse leads to a deterioration in the accuracy of determining the position of the maximum of the cross-correlation function, and the difference from the actual shift of the scales can reach several counts. The situation is expectedly aggravated if one of the pulses has a low $\mathrm{S} / \mathrm{N}$ value.

Once again, we specially note that the formal error of fitting the polynomial weakly depends on the S/N and broadening in the channel. Moreover, for wide pulses, the maximum of the cross-correlation function becomes smoother, and this even leads to a decrease in the formal inscribing error. Thus, for realistic estimates of accuracy, it is necessary to use the value of the actual deviation of the maximum from the position preset in the simulation.

\section{CONCLUSIONS}

The delay model is based on the difference between the coordinates of the lunar and terrestrial points in the barycentric frame of reference, considering the effects of signal propagation in the gravitational field of the Solar System and the curvature of the front. The model error is estimated at $\sim 30 \mathrm{~ns}$. The mutual course of the lunar and terrestrial time scales was calculated by numerical integration. It is shown that the amplitude of variations after subtracting the secular term is $\sim 150 \mu \mathrm{s}$. The size of the variations is such that they must be taken into account when synchronizing the scales.

The results of modeling the accuracy of the binding of the two scales fit into the expected ones. The pulse sampling rate should correspond to the pulse broadening in the channel. When the $\mathrm{S} / \mathrm{N}$ of one of the pulses deteriorates, the method continues to work, because another pulse with a high S/N ratio "pulls out" the correlation function, in which the position of the maximum is determined with sub-discrete accuracy. In units of time, with a sampling rate of $6 \mu$ s and $S / N>10$, the scale synchronization accuracy reaches $\sim 0.5 \mu \mathrm{s}$. 


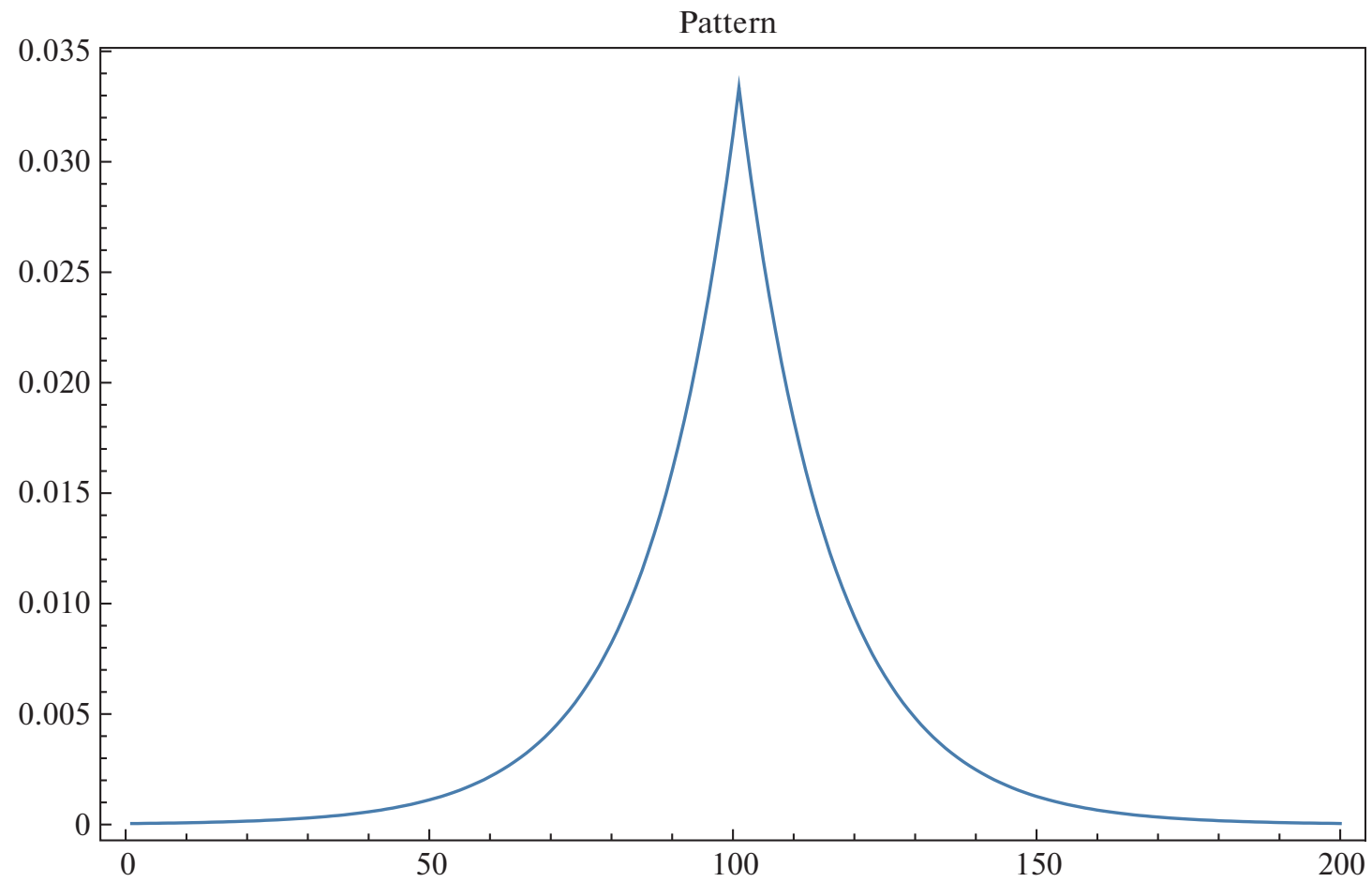

Fig. 7. Double exponential pattern for smoothing the cross-correlation function. The exponent is matched to the pulses of the pulsar PSR B0531+21.
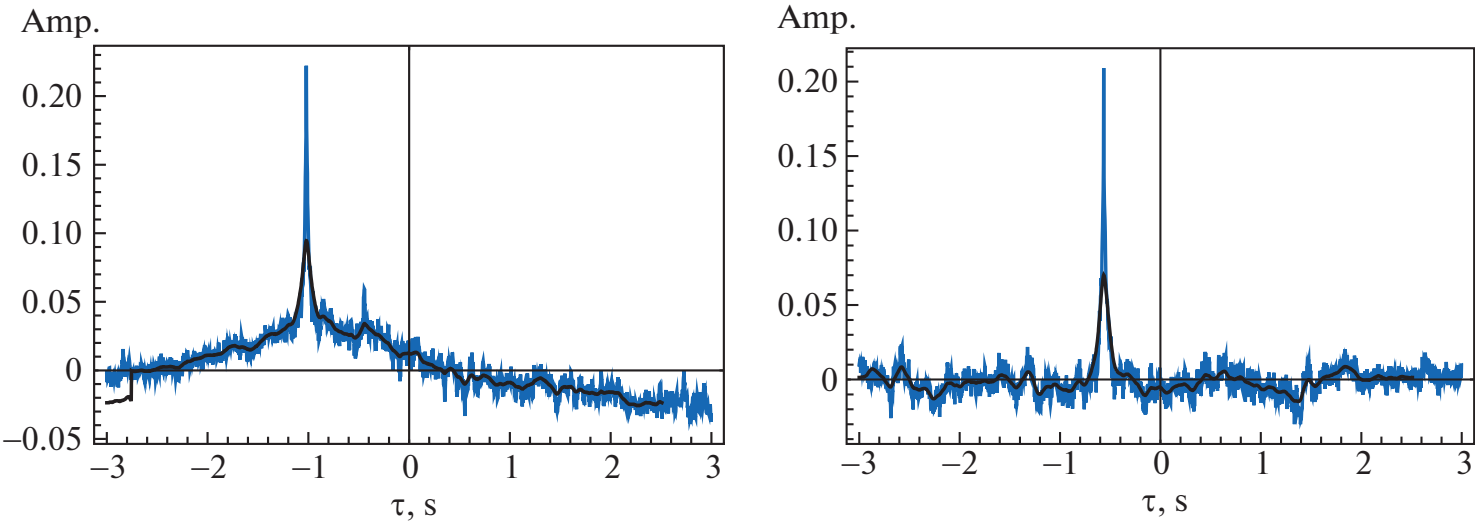

Fig. 8. Cross-correlation functions of the initial and noisy impulses. The function smoothed with an exponential window is shown in black. The shift is clearly visible -1.0211252 and -0.5640922 seconds, corresponding to the delay model.
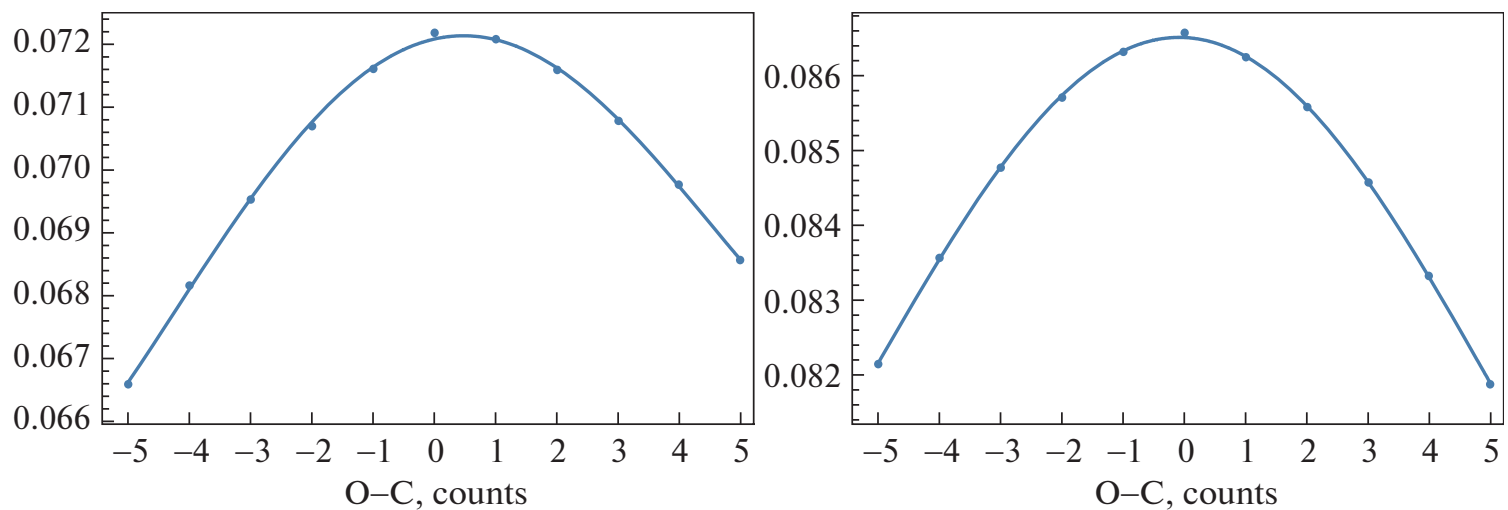

Fig. 9. Measurement of the maximum of the cross-correlation function by fitting two giant pulses with a polynomial. 
The situation worsens significantly if equipment with wide frequency channels is used, in which the pulse broadening is equal to several discretes. In this case, the comparison accuracy drops by more than an order of magnitude to $\sim 20 \mu \mathrm{s}$.

When using high frequencies (several gigahertz) and wide bands (several hundred megahertz), the potential accuracy of the synchronization of scales using the proposed method reaches a subnanosecond level at intervals of more than a day, which makes it possible to compare time scales on the bodies of the Solar System at the most modern level of accuracy. Due to the irregular and relatively rare GP tracking, the proposed method, in our opinion, is not suitable for consumers who need real-time coordinates and/or scale labels. It is better for them to use purely radiotechnical methods.

\section{ACKNOWLEDGMENTS}

The authors are grateful to V.A. Potapov for providing observational data with the BSA antenna of the Lebedev Physical Institute.

\section{OPEN ACCESS}

This article is licensed under a Creative Commons Attribution 4.0 International License, which permits use, sharing, adaptation, distribution and reproduction in any medium or format, as long as you give appropriate credit to the original author(s) and the source, provide a link to the
Creative Commons licence, and indicate if changes were made. The images or other third party material in this article are included in the article's Creative Commons licence, unless indicated otherwise in a credit line to the material. If material is not included in the article's Creative Commons licence and your intended use is not permitted by statutory regulation or exceeds the permitted use, you will need to obtain permission directly from the copyright holder. To view a copy of this licence, visit http://creativecommons.org/licenses/by/4.0/.

\section{REFERENCES}

1. Yu. P. Ilyasov, V. I. Kondrat'ev, V. V. Oreshko, M. V. Popov, and V. A. Soglasnov, Izmerit. Tekh., No. 10, 16 (2009).

2. T. H. Hankins, J. S. Kern, J. C. Weatherall, et al., Nature (London, U.K.) 422, 141 (2003).

3. M. Popov, V. Soglasnov, V. Kondratiev, A. Bilous, O. Moshkina,V. Oreshko, Yu. Ilyasov, M. Sekido, and T. Kondo, Publ. Astron. Soc. Jpn. 61, 1 (2009).

4. S. C. Lundgren, J. M. Cordes, M. Ulmer, S. M. Matz, S. Lomatch, R. S. Foster, and T. Hankins, Astrophys. J. 453, 433 (1995).

5. S. N. Sun, W. M. Yan, and N. Wang, Mon. Not. R. Astron. Soc. 501, 3900 (2021).

6. M. V. Popov, V. A. Soglasnov, V. I. Kondrat'ev, and A. V. Bilous, Astron. Rep. 52, 900 (2008).

7. A. E. Rodin, V. V. Oreshko, V. A. Potapov, M. S. Pshirkov, and M. V. Sazhin, Astron. Rep. 64, 499 (2020).

Translated by E. Seifina 